\title{
M680I/M694V Heterozygous Mutation in Early Onset Familial Mediterranean Fever
}

\author{
Widad Maha Darwish $^{\mathrm{a}}\left(\mathbb{D}\right.$, Sohaib Bassel Darwish ${ }^{\mathrm{b}} \mathbb{0}$, Muhammad Bassel Darwish ${ }^{\mathrm{C}}$, \\ Bassel Fayez Darwish ${ }^{\text {a, d }}$ (D)
}

\begin{abstract}
Familial Mediterranean fever (FMF) is a hereditary autoinflammatory disorder affecting individuals with biallelic pathogenic mutations in the MEFV gene. The disease is characterized by recurrent attacks of fever and serosal inflammation as manifested by abdominal and chest pain. This case report presents an FMF case with a 3-year history of pain crises consisting of severe abdominal pain and fever, lasting up to $72 \mathrm{~h}$. Genetic investigation identified an uncommon heterozygous mutation in the MEFV gene. This mutation is associated with a more severe phenotype of FMF and may lead to an early onset of the disease.
\end{abstract}

Keywords: Familial Mediterranean fever; Mutation; Heterozygous mutation; Fever

\section{Introduction}

Familial Mediterranean fever (FMF) is an autoinflammatory disorder that is usually considered an autosomal recessive disease [1]. It is characterized by attacks of fever and serosal inflammation [2]. FMF predominantly affects Mediterranean people, including Arabs, Turks, Armenians, Jews, and individuals of North African descent [3]. The underlying mechanism by which FMF develops is genetic [4, 5]. Mediterranean fever (MEFV) is a gene located on the short arm of chromosome 16 and is responsible for coding for the protein pyrin, which has an important role in the innate immune system $[1,6,7]$. In FMF, MEFV is mutated and leads to symptoms [1, 6]. Symptoms present in the form of attacks that are recurrent, painful, and

Manuscript submitted July 11, 2021, accepted July 27, 2021

Published online August 25, 2021

${ }^{a}$ Health Call Clinic, Dubai Healthcare City, Dubai, UAE

${ }^{b}$ College of Medicine, Mohammed Bin Rashid University, Dubai Healthcare City, Dubai, UAE

'Department of Surgery, Valley Health System, Las Vegas, NV, USA

${ }^{\mathrm{d} C}$ Corresponding Author: Bassel Fayez Darwish, Health Call Clinic, Dubai

Healthcare City, Dubai 505032, UAE.

Email: dubairheumatologist@gmail.com

doi: https://doi.org/10.14740/jmc3747 characterized by fever, abdominal pain, chest pain, and joints pain with a duration that varies considerably even in the same patient $[8,9]$. Patients are asymptomatic between attacks, and there does not appear to be a specific triggering factor for the attacks [10]. Left untreated, repeated bouts of inflammation can lead to secondary amyloidosis, which may cause systemic amyloid A protein deposition, notably in the kidneys [11]. Colchicine is the treatment of choice and can significantly reduce the severity of attacks [12-17].

This report presents a case of FMF in a three-and-a-halfyear-old girl of East European and North African Arab descent with an uncommon heterozygous MEFV mutation.

\section{Case Report}

\section{Investigations}

A three-and-a-half-year-old girl was brought to the clinic by her parents due to musculoskeletal pain, particularly knee pain, and recurrent pain crises consisting of sudden, severe, generalized abdominal pain and fever lasting $12-16 \mathrm{~h}$. The patient started experiencing these attacks at the age of 6 months, initially occurring every $1-2$ months and lasting $16-24 \mathrm{~h}$, and have increased in frequency and progressed to weekly episodes lasting up to $72 \mathrm{~h}$. The last attack was by far the longest, lasting $72 \mathrm{~h}$, but subsided prior to presentation.

The patient is a carrier of beta-thalassemia trait but has no other significant medical or surgical history. The mother reported that the patient developed reactions including difficulty breathing to dimethindene, cetirizine, cefuroxime, and amoxicillin/clavulanate. Her mother is a carrier for beta-thalassemia trait. Her father has no known medical conditions. There is no known family history of FMF. The mother is of East European descent and the father is of North African Arab descent.

\section{Diagnosis}

Physical examination was unremarkable and the patient was afebrile. Labs obtained on presentation revealed an erythrocyte sedimentation rate of $52 \mathrm{~mm} / \mathrm{h}$, blood leukocytes of $17,100 /$ $\mu \mathrm{L}$, a positive C-reactive protein at $185 \mathrm{mg} / \mathrm{L}$, hemoglobin of $9.7 \mathrm{~g} / \mathrm{dL}$, mean corpuscular volume of $59 \mathrm{fL}$, platelets of $376,000 / \mathrm{mm}^{3}$ and urinalysis positive for ketones. A diagnosis 
of FMF was made based on the Tel Hashomer criteria and the patient was started on colchicine $0.5 \mathrm{mg}$ once daily.

Ethylenediaminetetraacetic acid (EDTA) whole blood sample was sent for genetic analysis at Eurofins Clinical Diagnostics, France. Genome Reference Consortium Human Build 37 (hg19) and NCBI Reference Sequence: NM_000243.2 were used as references. Next-generation sequencing (NGS) MEFV exon 1-10 and MiSeq (ILLUMINA) analyses were carried out and analyzed using the MiSeq Reporter 2.5.1 and Variant Studio 3.0 data analyzers. Mutations were confirmed with Sanger sequencing exon 10 and ABI 3130 XL. Results revealed two pathogenic missense variants identified in the MEFV gene in a heterozygous state: c.2080A $>$ G (p.Met694Val) and c.2040G $>$ A (p.Met680Ile). This suggests that the patient is compound heterozygous for these variants and is consistent with FMF.

\section{Treatment}

The patient continued taking colchicine $0.5 \mathrm{mg}$ once daily. However, a week later, she experienced another attack of pain similar in intensity to previous attacks and the colchicine dose was increased to $0.5 \mathrm{mg}$ twice daily. She responded well to the higher dose and did not develop any adverse effects. Subsequent attacks were milder with only mild fever, mild knee pain, and no abdominal pain.

\section{Follow-up and outcomes}

The patient was lost to follow up after the last increase in dose and improvement in symptoms.

\section{Discussion}

This case illustrates the importance of considering FMF as a differential diagnosis in a child presenting with fever, joint pain, and abdominal pain. FMF should be considered even at a very young age, as seen in this patient who started experiencing attacks at the age of 6 months but was only diagnosed 3 years later. The diagnosis is primarily clinical and should not be delayed by genetic testing to confirm the diagnosis. Starting the appropriate treatment is affordable, safe, effective, and provides immediate relief for the patient while reducing the risk of long-term complications such as secondary amyloidosis.

FMF is caused by gain of function mutations in the MEFV gene located on chromosome 16 [18]. MEFV gene encodes pyrin, a protein which through interaction with GTPases results in inflammasome activation and modulates production of interleukin-1 $\beta$ (IL-1 $\beta$ ) and in turn prevents inappropriate inflammatory reactions $[19,20]$. An increase in IL-1 $\beta$ and IL-18 causes cells to undergo pyroptosis, a process of inflammatory death [18]. The rise in pro-inflammatory cytokines gives rise to the inflammatory presentation seen in FMF patients. More than a hundred mutations have been identified in the MEFV gene, the five accounting for most FMF cases being V726A,
M694V, M694I, M860I, and E148Q [20]. The heterozygous MEFV mutation combination diagnosed in this case is uncommon and is associated with a more severe phenotype of FMF.

In this case, the patient met the clinical criteria for the diagnosis of FMF [9]. The Tel Hashomer criteria are the most commonly used criteria for diagnosis of FMF in adults and consist of four major, five minor, and 10 supportive criteria [9, 21]. The major criteria include generalized peritonitis, unilateral pleuritis, monoarthritis (hip, knee, ankle), and fever alone [9]. The minor criteria include incomplete attacks, exertional leg pain, and a favorable response to colchicine [9, 21]. A diagnosis of FMF is made when $\geq 1$ major criteria are present, $\geq$ 2 minor criteria are present, one minor criterion plus $\geq 5$ supportive criteria, or one minor criterion plus $\geq 4$ of the first five supportive criteria [9]. The Tel Hashomer criteria yield a high sensitivity of $99 \%$ but a relatively low specificity of $55 \%$ and are successful in diagnosing FMF in childhood [21, 22]. In this case, the patient presented with three major (abdominal pain, joint pain, and fever), one minor (favorable response to colchicine) criterion, and five supportive criteria (appropriate ethnic origin, age $<20$ years at disease onset, severe attacks requiring bed rest, spontaneous remission of attacks, and symptom-free intervals). The patient had never received colchicine prior to presentation.

FMF is primarily a clinical diagnosis. Inflammatory markers can be increased during attacks but are non-specific. The Tel Hashomer criteria include laboratory findings such as inflammatory markers and urinary findings [18]. Transient inflammatory response, with $\geq 1$ abnormal white blood cell (WBC) count test results, erythrocyte sedimentation rate, serum amyloid A, fibrinogen, and episodic proteinuria/hematuria are considered supportive criteria in the diagnosis of FMF [9]. There are no specific tests for diagnosing FMF apart from genetic testing.

Mutations in FMF patients are usually found throughout the MEFV gene. The most severe forms of the disease are associated with M694V and M680I mutations which are clustered in exon 10 of the gene. The incidence of the Met6801le/ Met694Val heterozygous mutation is uncommon, with incidence rates ranging from $0.79 \%$ to $1.9 \%$ in FMF patients as reported by previous FMF studies $[23,24]$. It appears that patients with homozygous Met694V mutation tend to have an earlier onset of FMF, although there is conflicting evidence [25].

The first-line management for FMF is prophylactic colchicine regardless of the frequency or intensity of attacks. The mechanism of action of colchicine in FMF is not entirely understood. However, it is known that colchicine prevents microtubule elongation by binding to tubulin monomers and inhibiting polymer formation. Thus, the organization of actin cytoskeleton could be the link between pyrin and colchicine [26]. The starting dose is $\leq 0.5 \mathrm{mg} /$ day for children $<5$ years of age, $0.5-1 \mathrm{mg} /$ day for children 5 - 10 years of age, and $1-1.5 \mathrm{mg} /$ day in children $>10$ years of age and adults [26]. The most common side effects are gastrointestinal, including diarrhea $[18,26]$.

Colchicine has been shown to prevent the complication of secondary amyloidosis in patients diagnosed with FMF [19]. Use of intermittent high-dose colchicine only for treat- 
ment of acute attacks is not recommended as it does not protect from amyloidosis resulting from low-grade inflammation during asymptomatic periods. Colchicine treatment is lifelong in FMF [26]. Anti-interleukin-1 drugs are used in colchicine resistant or intolerant FMF cases because of pyrin's role in the regulation of IL-1 $\beta$ activation [26]. With early diagnosis and prompt treatment, FMF has an excellent prognosis [18].

\section{Conclusion}

FMF is a genetic autoinflammatory disorder that needs to be considered in the differential of a patient presenting with generalized symptoms such as fever and abdominal pain, including patients at a very young age. Family history and ethnic descent can help aid the clinician in the diagnosis. Diagnosis should not be delayed by genetic testing. M680I/M694V mutations produce more severe symptoms but do not alter the treatment of choice. Prompt diagnosis and initiation of treatment are crucial in reducing the patient's symptoms and reducing the risk of long-term complications.

\section{Acknowledgments}

The authors thank Eurofins Biomnis, Eurofins Clinical Diagnostics, France for their help in analyzing the genetic testing results.

\section{Financial Disclosure}

None related to this project.

\section{Conflict of Interest}

None related to this project.

\section{Informed Consent}

Not applicable.

\section{Author Contributions}

All authors certify that he or she has participated sufficiently in the intellectual content, the analysis of data. Each author has reviewed the final version of the manuscript and approves it for publication. Should the editors request the data upon which the work is based, the authors shall produce it.

\section{Data Availability}

The authors declare that data supporting the findings of this study are available within the article.

\section{References}

1. French FMFC. A candidate gene for familial Mediterranean fever. Nat Genet. 1997;17(1):25-31.

2. Ben-Chetrit E, Touitou I. Familial mediterranean Fever in the world. Arthritis Rheum. 2009;61(10):1447-1453.

3. Touitou I. The spectrum of Familial Mediterranean Fever (FMF) mutations. Eur J Hum Genet. 2001;9(7):473-483.

4. Grossman C, Kassel Y, Livneh A, Ben-Zvi I. Familial Mediterranean fever (FMF) phenotype in patients homozygous to the MEFV M694V mutation. Eur J Med Genet. 2019;62(6):103532.

5. Shinar Y, Livneh A, Langevitz P, Zaks N, Aksentijevich I, Koziol DE, Kastner DL, et al. Genotype-phenotype assessment of common genotypes among patients with familial Mediterranean fever. J Rheumatol. 2000;27(7):1703-1707.

6. Ancient missense mutations in a new member of the RoRet gene family are likely to cause familial Mediterranean fever. The International FMF Consortium. Cell. 1997;90(4):797-807.

7. Stehlik C, Reed JC. The PYRIN connection: novel players in innate immunity and inflammation. J Exp Med. 2004;200(5):551-558.

8. Sohar E, Gafni J, Pras M, Heller H. Familial Mediterranean fever. A survey of 470 cases and review of the literature. Am J Med. 1967;43(2):227-253.

9. Livneh A, Langevitz P, Zemer D, Zaks N, Kees S, Lidar T, Migdal A, et al. Criteria for the diagnosis of familial Mediterranean fever. Arthritis Rheum. 1997;40(10):18791885.

10. Lidar M, Livneh A. Familial Mediterranean fever: clinical, molecular and management advancements. Neth J Med. 2007;65(9):318-324.

11. Ozen S. Renal amyloidosis in familial Mediterranean fever. Kidney Int. 2004;65(3):1118-1127.

12. Goldfinger SE. Colchicine for familial Mediterranean fever. N Engl J Med. 1972;287(25):1302.

13. Zemer D, Revach M, Pras M, Modan B, Schor S, Sohar $\mathrm{E}$, Gafni J. A controlled trial of colchicine in preventing attacks of familial mediterranean fever. N Engl J Med. 1974;291(18):932-934

14. Dinarello CA, Wolff SM, Goldfinger SE, Dale DC, Alling DW. Colchicine therapy for familial mediterranean fever. A double-blind trial. N Engl J Med. 1974;291(18):934937.

15. Goldstein RC, Schwabe AD. Prophylactic colchicine therapy in familial Mediterranean fever. A controlled, double-blind study. Ann Intern Med. 1974;81(6):792794.

16. Wright DG, Wolff SM, Fauci AS, Alling DW. Efficacy of intermittent colchicine therapy in familial Mediterranean fever. Ann Intern Med. 1977;86(2):162-165.

17. Zemer D, Livneh A, Danon YL, Pras M, Sohar E. Longterm colchicine treatment in children with familial Mediterranean fever. Arthritis Rheum. 1991;34(8):973-977.

18. Tufan A, Lachmann HJ. Familial Mediterranean fever, from pathogenesis to treatment: a contemporary review. 
Turk J Med Sci. 2020;50(SI-2):1591-1610.

19. Migita K, Asano T, Sato S, Koga T, Fujita Y, Kawakami A. Familial Mediterranean fever: overview of pathogenesis, clinical features and management. Immunol Med. 2018;41(2):55-61.

20. Yepiskoposyan L, Harutyunyan A. Population genetics of familial Mediterranean fever: a review. Eur J Hum Genet. 2007;15(9):911-916.

21. Yalcinkaya F, Ozen S, Ozcakar ZB, Aktay N, Cakar N, Duzova A, Kasapcopur O, et al. A new set of criteria for the diagnosis of familial Mediterranean fever in childhood. Rheumatology (Oxford). 2009;48(4):395-398.

22. Kondi A, Hentgen V, Piram M, Letierce A, GuillaumeCzitrom S, Kone-Paut I. Validation of the new paediatric criteria for the diagnosis of familial Mediterranean fever: data from a mixed population of 100 children from the
French reference centre for auto-inflammatory disorders. Rheumatology (Oxford). 2010;49(11):2200-2203.

23. Bagheri M, Rad IA. Analysis of the most common three MEFV mutations in 630 patients with familial Mediterranean fever in Iranian Azeri Turkish population. Maedica (Bucur). 2017;12(3):169-173.

24. Procopio V, Manti S, Bianco G, Conti G, Romeo A, Maimone F, Arrigo T, et al. Genotype-phenotype correlation in FMF patients: A "non classic" recessive autosomal or "atypical" dominant autosomal inheritance? Gene. 2018;641:279-286.

25. Shohat M, Halpern GJ. Familial Mediterranean fever-a review. Genet Med. 2011;13(6):487-498.

26. Ozen S, Batu ED, Demir S. Familial Mediterranean fever: recent developments in pathogenesis and new recommendations for management. Front Immunol. 2017;8:253. 